doi: http://dx.doi.org/10.7124/bc.0009FB

\section{S-7. Life time of some products of human rDNA intergenic spacer.}

T. Vacik, S. Kereïche, I. Raska, D. Cmarko, E. Smirnov

Charles University, Academic esmir@lf1.cuni.cz

In human cells, ribosomal genes are interspersed by $30 \mathrm{~kb}$ long intergenic spacers (IGS). Recently it has been found that all, or almost all, parts of IGS may be transcribed, and at least some of them play important role in the regulation of rDNA transcription, maintenance of nucleolar architecture and reaction of the cell nucleus to the stress. But, since each cell contains hundreds not quite identical copies of IGS, the structure and functions of this locus remain poorly understood, dynamics of its products has not been studied specially. In this study we used qPCR to measure expression levels of various ribosomal and spacer regions at different times after inhibition of the transcription by ActD. This approach allowed us to measure real or extrapolated halflife times of some IGS loci. Our study reveals characteristic dynamic patterns suggestive of various pathways of RNA utilization and decay.

doi: http://dx.doi.org/10.7124/bc.0009FC

\section{S-8. Mechanisms of telomere instability in the Drosophila female germline}

O. Sokolova ${ }^{1}$, A. Gonchar ${ }^{1,2}$, A. Kalmykova ${ }^{1}$ ${ }^{1}$ Institute of Molecular Genetics, Russian Academy of Sciences, Moscow 123182, Russia; ${ }^{2}$ Department of Biochemistry, Faculty of Biology, Lomonosov Moscow State University, 119991 Moscow, Russia sokolova@img.ras.ru

Telomeres are nucleoprotein complexes that protect the ends of eukaryotic linear chromosomes from degradation and fusion. Telomere dysfunction leads to developmental disorders, oncogenesis and aging. A telomere consists of DNA repeats and their bound proteins, as well as a telomeric RNA that is transcribed from telomeric repeats. Transcription of telomeric repeats is a conserved feature of telomeres in all studied species. Telomeric transcripts are maintained at a low level but their significant fraction is retained near telomeres. The telomeres of Drosophila are maintained in the absence of telomerase, by the transpositions of the specialized telomeric non-LTR retrotransposons; the HeT-A element being the most abundant. Here, we address a question on the factors of telomere instability in the Drosophila female germline. The level of HeT-A RNA in the germline is regulated by Piwi-interacting (pi) RNAs, the nuclear RNA surveillance system and transcription factors. Depletions of any of these components leading to the upregulation of HeT-A expression cause early embryonic lethality. It is suggested that accumulation of chromatin-bound telomeric RNA can destabilize telomeric DNA, leading to DNA lesions and recombination events. To find out more about a link between telomeric RNA abundance and telomere instability, we performed a study of the telomere integrity when the telomeric RNA biogenesis was impaired, which led to HeT-A overexpression. Moreover, abundant telomeric transcripts were revealed in a specific RNA fraction associated with chromatin. We detected the presence of 